\title{
Traslado aéreo de un paciente con insuficiencia cardíaca conectado a ECMO
}

\author{
Javier Labbé ${ }^{1}$, Nicolás Drolett ${ }^{2}$, Manuel Quiroz ${ }^{3}$, Camila Vallejo ${ }^{4}$, Oneglio Pedemonte. ${ }^{5}$ \\ 1. Cirujano Cardiovascular, Jefe de unidad, Cirugía Cardiaca, Hospital Clínico Regional de Antofagasta Dr. Leonardo Guzmán, \\ Antofagasta, Chile. \\ 2. Estudiante de Medicina, Facultad de Medicina y Odontología, Universidad de Antofagasta, Antofagasta, Chile. \\ 3. Residente de Cirugía, Hospital Clínico San Borja-Arriarán, Universidad de Chile, Santiago, Chile. \\ 4. Médico Cirujano, Unidad de Cirugía Cardíaca, Hospital Regional de Antofagasta Dr. Leonardo Guzmán, Antofagasta, Chile. \\ 5. Unidad de paciente crítico cardiovascular, Hospital Gustavo Fricke, Universidad de Valparaíso, Viña del Mar, Chile.
}

\section{Resumen:}

El traslado aéreo con ECMO es una forma segura y eficaz de trasladar pacientes para obtener mejores opciones terapéuticas. Este caso clínico ilustra la factibilidad, de este modo de apoyo al tratamiento de pacientes críticos, en este caso de una valvulopatía con indicación quirúrgica.

\section{Air transfer of a heart failure patient on ECMO}

Air transfer of patients on ECMO is a safe mode to allow for treatment of patients located in places with limited resources for intensive care treatments in cardiovascular disease. The present case illustrates the feasibility and conditions required to implement the procedure. The case herein reported was a patient with severe valvular heart disease requiring surgery

Key words: Cardiac surgery, extra corporeal membrane oxygenator, inter hospital transfer 


\section{Introducción}

La oxigenación con membrana extracorpórea (ECMO) es uno de los dispositivos de soporte cardiorrespiratorio más utilizado en los últimos 20 años ${ }^{1}$. Este equipo promueve el soporte hemodinámico y respiratorio gracias a una bomba y un oxigenador. Está indicado en caso de falla respiratoria, cardíaca o mixta, disminuyendo el daño por hipoperfusión y mejorando la sobrevida ${ }^{2}$.

El ECMO puede considerarse como un puente para tomar decisiones, especialmente cuando las opciones terapéuticas están fuera del centro hospitalario de ingreso ${ }^{3}$. El traslado aéreo en ECMO está reservado a pacientes críticos y cuyo centro de referencia está a una distancia considerable. El traslado de un paciente inestable a un centro que permita conectarlo a ECMO es una estrategia con alta mortalidad; debido a esto, se plantea como alternativa instalar el ECMO y luego realizar el traslado inter-hospitalario ${ }^{4}$.

En el norte de Chile, la escasez de unidades de cardiocirugía obliga a trasladar los pacientes a una distancia cercana a $1.200 \mathrm{~km}$ aproximadamente a nuestro centro de referencia en Viña del Mar ${ }^{5}$. Es por esto que el traslado de pacientes en avión ambulancia se ha hecho cada vez más común.

Existe escasa evidencia sobre traslados aéreos en pacientes con ECMO en la literatura nacional. Presentamos el caso de un paciente que presenta hipertensión pulmonar (HTP) grave secundaria a una valvulopatía mitral que luego de ser intervenido se traslada en avión ambulancia al Hospital Gustavo Fricke (HGF) bajo ECMO.

\section{Presentación del caso}

Hombre de 35 años, de nacionalidad boliviana, con antecedentes de valvulopatía mitral y arritmia completa por fibrilación auricular en tratamiento con anticoagulantes orales desde hace 7 años Seis meses antes del ingreso, coincidiendo con abandono del tratamiento, presenta disnea con clase funcional que llega a IV, asociado a edema de extremidades inferiores, palpitaciones, tos y disnea paroxística nocturna. A su ingreso en el Hospital Carlos Cisternas de Calama, se constató disneico e hipotenso. $\mathrm{Al}$ examen físico tenía ritmo irregular con soplo holosistólico en foco mitral grado III/VI, murmullo pulmonar disminuido globalmente en hemitórax derecho y reflujo hepatoyugular positivo. El electrocardiograma no evidenció infarto agudo al miocardio; la radiografía de tórax mostró cardiomegalia y derrame pleural derecho, Además, presentaba una insuficiencia renal aguda.

Se trasladó al paciente a la unidad de tratamiento inten- sivo (UTI) con diagnóstico de insuficiencia cardíaca descompensada y edema pulmonar agudo (EPA) y se inició manejo médico. Debido a la mala respuesta, se trasladó al Hospital Regional de Antofagasta (HRA) donde ingresó con diagnóstico de EPA y una infección urinaria baja tratada con ceftriaxona. El ecocardiograma reveló hipertensión pulmonar (HTP) grave, estenosis mitral cerrada y dilatación de aurícula izquierda, por lo cual se planificó el traslado al Hospital Gustavo Fricke para cirugía de recambio valvular.

Debido a la demora en la gestión del traslado se decidió estabilizar al paciente y realizar la intervención en el HRA. Se efectuó un recambio valvular mitral, observando una válvula mitral estrecha, calcificada, con músculos papilares hipertróficos y fusionados con los velos. Se implantó una prótesis Saint Jude ${ }^{\circledR}$, se logró salir de bomba por 30 minutos, pero al decanular la aorta disminuyeron las presiones y la saturación cerebral de oxígeno, y finalmente se decidió conectar el paciente a ECMO.

El paciente ingresó a UTI dependiente de drogas vaso-activas y, ante la falta de prostaglandinas y óxido nítrico en el HRA para la reducción de la HTP, se prepara el traslado del paciente en avión ambulancia asistido con ECMO al HGF en Viña del Mar (Fotos 1 y 2). Se logró el traslado con un buen funcionamiento de los sistemas de energía y gases sin presentar complicaciones (Fotos 3 y 4 ).

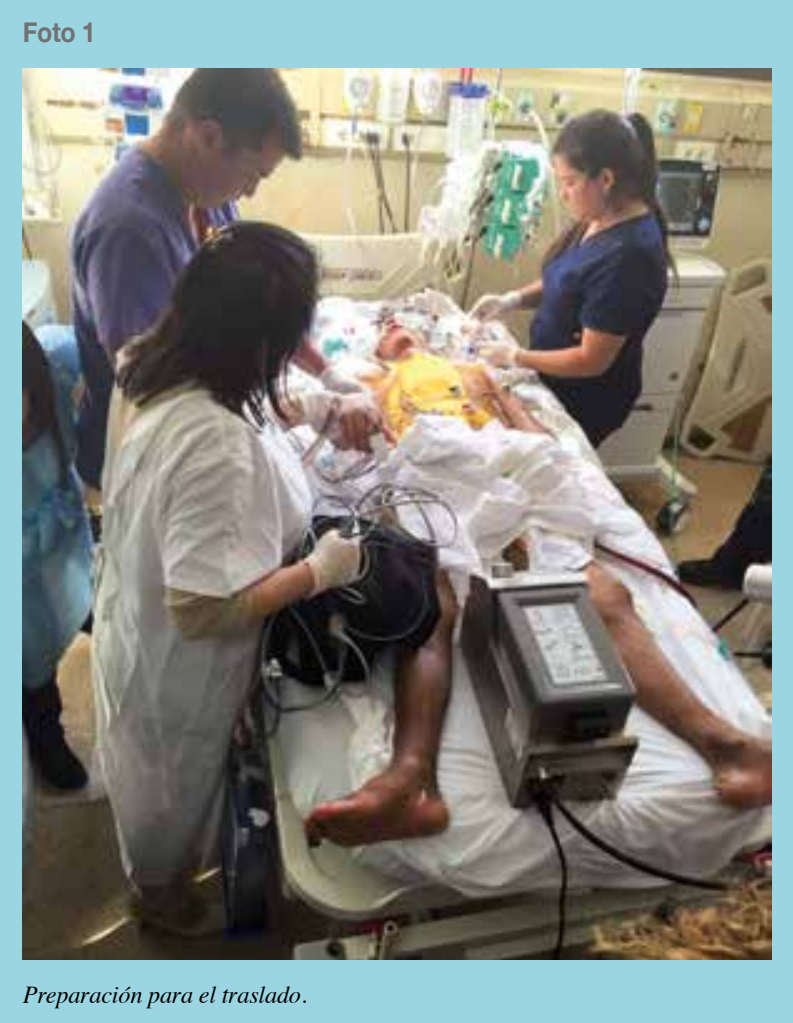



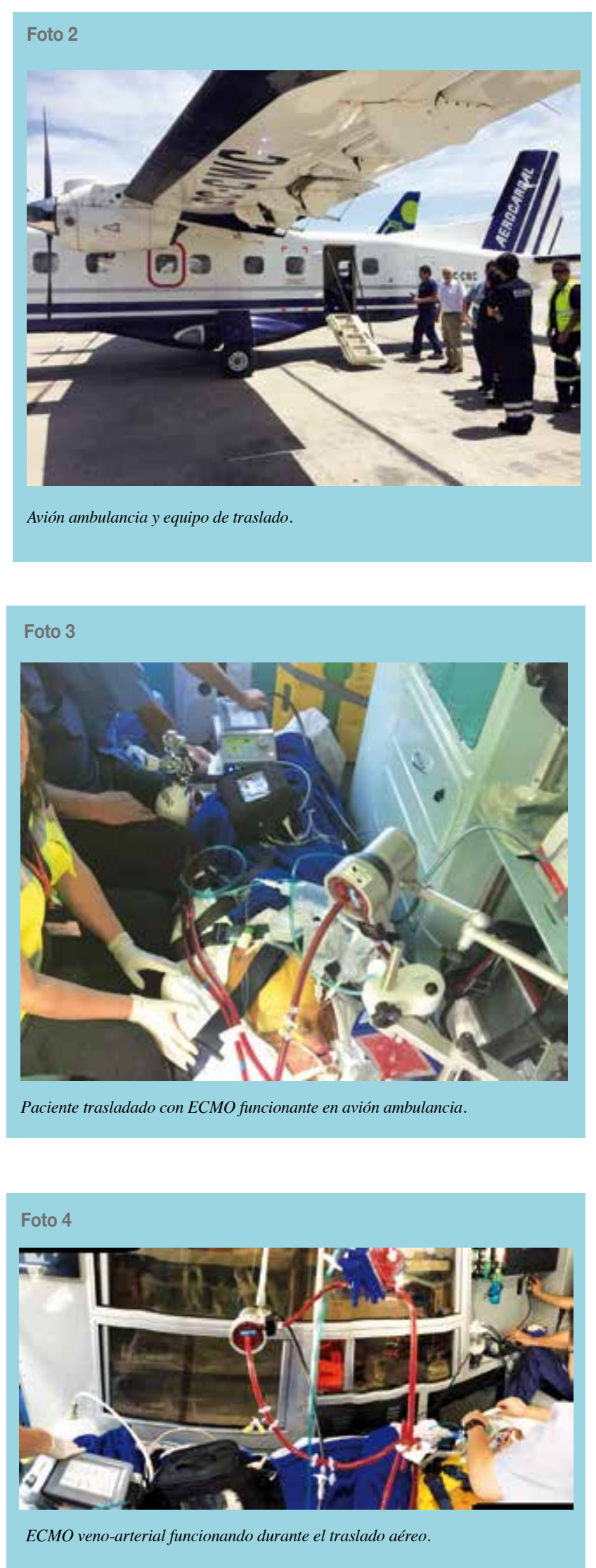

Durante su estadía de 14 días en el HGF el paciente sufrió una sepsis producto de una neumonía intrahospitalaria y falleció.

\section{Discusión}

El trasporte inter-hospital es utilizado cuando la capacidad diagnóstica o terapéutica del hospital de origen es limitada ${ }^{7}$. La regionalización de unidades mejor equipadas a lo largo de los países ha contribuido a la utilización cada vez más frecuente de los traslados inter-hospitalarios ${ }^{8}$. Es fundamental evaluar con criterio los beneficios de un traslado y asegurarse de que estos sean superiores a las desventajas, coordinar de manera eficiente y rápida el traslado con el equipo correspondiente en el hospital receptor ${ }^{9}$.

Desde el año 2012 el HRA con el apoyo del Hospital Gustavo Fricke y la Fundación Kaplan, crearon la unidad de Cirugía Cardiovascular del sistema público de salud correspondiente a la macro zona norte, para brindar a los pacientes una opción más expedita al tratamiento de las patologías quirúrgicas cardiovasculares. Sin embargo, para nuestra zona la distancia a este centro es considerable ${ }^{11}$.

Respecto a las ventajas del traslado aéreo versus el traslado terrestre Stone y cols., (1994) ${ }^{12}$ no encontraron ventajas significativas en pacientes cardíacos, pero este estudio no incluía distancias equivalentes a la que reportamos en este caso. Por otro lado, la literatura señala ventajas en cuanto al tiempo de traslado y menor estadía hospitalaria ${ }^{6,13-15}$. Los traslados aéreos generalmente ocurren en un contexto de urgencia, por lo que es frecuente el uso de dispositivos de apoyo como el $\mathrm{ECMO}^{4,11}$. Este recurso permite mantener estable al paciente y minimizar el daño tisular, mientras se decide el plan de tratamiento ${ }^{2}$.

En la literatura internacional destaca la serie de Broman y cols (2015), transportando 282 pacientes a distancias entre 6.900 y $13.000 \mathrm{~km}$. La mayoría de los traslados fueron realizados por vía aérea. El 22\% de los pacientes sufrió algún tipo de complicación relacionada con su patología, pero no hubo mortalidad ${ }^{16}$. Vaja y cols., (2015) ${ }^{17}$ comunican 102 pacientes, recorriendo distancias cercanas a los $400 \mathrm{~km}$. Un total de 22 de estos pacientes fueron transportados por vía aérea, y entre las complicaciones destaca un paciente que presentó una taquicardia ventricular, la cual fue revertida. Roger y cols $(2013)^{18}$ por su parte entregan datos similares, transportando 7 pacientes en helicóptero y 8 en avión sin presentar complicaciones ni mortalidad ${ }^{18}$. 
La experiencia nacional publicada es escasa. Díaz $(2011)^{4}$ presenta una serie de 11 pacientes trasladados en ECMO, 9 en ambulancia y 2 en avión; 4 de estos pacientes tenían indicación de ECMO por causa cardíaca con sobrevida de un paciente. Esta serie no presenta complicaciones en el traslado, sin embargo, menciona problemas logísticos, entre ellos la autonomía de los gases y la electricidad ${ }^{2}$. En nuestra experiencia estos problemas fueron sorteados mediante la revisión constante de la electricidad disponible en el avión y la batería del ECMO, la cual tiene una autonomía de 30 minutos. Respecto a los gases contábamos con una autonomía suficiente considerando la duración de nuestro viaje. Utilizamos un avión ambulancia y un equipo integrado por un cirujano cardiovascular, un médico cirujano, un enfermero y 2 paramédicos.

En conclusión, el traslado aéreo de pacientes en ECMO es eficiente y seguro, permite la posibilidad de tratar pacientes que en su centro no tienen opción terapéutica. Es importante la coordinación entre el personal, la revisión de los equipos y la evaluación exhaustiva del paciente antes y durante el traslado para minimizar las complicaciones. 


\section{Referencias}

1. NAPOLITANO L. Nonventilatory strategies for patients with life-threatening $2009 \mathrm{H} 1 \mathrm{~N} 1$ influenza and severe respiratory failure. Crit Care Med 2010; 38: 74-90.

2. HANSELL D. ECLS equipments and devices. En: Van Meurs K, Lally K P, Peek G, Zwischenberger J. ECMO Extracorporeal Cardiopulmonary Support in Critical Care. 3rd Edition, Ann Arbor, MI, 2005. Extracorporeal life support organization, pp: 107-19.

3. JOHN R. Experience with the Levitronix CentriMag circulatory support system as a bridge to decision in patients with refractory acute cardiogenic shock and multisystem organ failure. J Thorac Cardiovasc Surg. 2007; 134: 351-3.

4. DÍAZ R. ECMO y ECMO Mobile. Soporte Cardio Respiratorio Avanzado. Rev Med Clin Condes. 2011; 22: 377-87.

5. SERVICIO DE SALUD ANTOFAGASTA. Balance positivo primer año de cirugías cardíacas en Antofagasta. Red Salud [online] 2013. Disponible en: http://ssa.redsalud.gob. $\mathrm{cl} / \mathrm{p}=1559$ [consultado el 07-06-2015].

6. BERNS KS, HANKINS DG, ZIETLOW SP. Comparison of air and ground transport of cardiac patients. Air Med J. 2001; 20: $33-6$.

7. GRAY A, GILL S, AIREY M, WILLIAMS R. Descriptive epidemiology of adult critical care transfers from the emergency department. Emerg Med J. 2003; 20: 242-6.

8. WAGNER J, IWASHYNA TJ, KAHN JM. Reasons underlying interhospital transfers to an academic medical intensive care unit. J Crit Care. 2013; 28: 202-8.

9. SINGH JM, MACDONALD RD. Pro/con debate: Do the benefits of regionalized critical care delivery outweigh the risks of interfacility patient transport?. Crit Care. 2009; 13: 219.

10. SETHI D., SUBRAMANIAN S. When place and time matter: How to conduct safe inter-hospital transfer of patients. Saudi
J Anaesth. 2014; 8: 104-13.

11. LABBE J, QUIROZ M, DROLETT N. Traslado aéreo con balón de contrapulsación aórtico de paciente con ruptura del septum interventricular post infarto al miocardio. Rev Chil Cardiol. 2014; 33: 228-33.

12. STONE CK, HUNT RC, SOUSA JA, WHITLEY TW, THOMAS SH. Interhospital transfer of cardiac patients: does air transport make a difference?. Air Med J. 1994; 13: 159-62.

13. SVENSON JE, O'CONNOR JE, LINDSAY MB. Is air transport faster? A comparison of air versus ground transport times for interfacility transfers in a regional referral system. Air Med J. 2006; 25: 170-2.

14. MYERS J, PSIRIDES A, HATHAWAY K, LARSEN P. Air transport by the Wellington Flight Service: a descriptive analysis of interhospital transfers over a 5 year period in the Wellington region of New Zealand. N Z Med J. 2012; 125. Diesponible en: http://journal.nzma.org.nz/journal/125-1351/5103 [consultado el 09-08-2016].

15. HOLLAND J, COOKSLEY DG. Safety of helicopter aeromedical transport in Australia: a retrospective study. Med J Aust. 2005; 182: 17-19.

16. BROMAN LM, HOLZGRAEFE B, PALMÉR K, FRENCKNER B. The Stockholm experience: interhospital transports on extracorporeal membrane oxygenation. Critical Care. 2015; 19: 278.

17. VAJA R, CHAUHAN I, JOSHI V, SALMASI Y, PORTER R, FAULKNER G, et al. Five-year experience with mobile adult extracorporeal membrane oxygenation in a tertiary referral center. Journal of Critical Care. 2015; 30: 1195-8.

18. ROGER D, DUDOUIT JM, RÉSIÈRE D, MEHDAOUI H, COURCIER D, VILLAIN L, et cols. Interhospital transfer of ECMO-assisted patients in Martinique. Ann Fr Anesth Reanim. 2013 ; 32: 307-14. 